\title{
Molecular Modelling and Insilico Engineering of PapMV-CP Towards Display and Development of Capripox Viral Like Particles Based on Immunogenic P32 Envelop Protein is the Homologous of the Vaccinia-Viral H3L Gene: An Insilico Approach
}

\author{
Burranboina Kiran Kumar ${ }^{1}$ (D) K. M. Kumar ${ }^{1,3}$ - G. B. Manjunatha Reddy ${ }^{2}$ Sunil Abraham ${ }^{4} \cdot$ R. Yogisharadhya $^{2}$. \\ C. N. Prashantha ${ }^{1}$
}

Accepted: 22 December 2019 / Published online: 6 January 2020

๑) Springer Nature B.V. 2020

\begin{abstract}
Viral-like particles are assembled from capsid protein structural subunits of different viruses and have ability to establish research in biomedicals, like construction of novel safety vaccines, gene therapy vectors by delivering systems for nucleic acids, small biomolecules and diagnostics. Papaya Mosaic Viral nanoparticals can provide as a vaccine candidate helps to increase the immunity by fusing the epitope based peptide antigen. Capripox viruses are the genus comprises Lymphy skin-disease, Sheep and Goat pox Viruses are notified by The World Animal Health Organization (OIE) based on their economic impotence act as a transboundary animal diseases viruses of sheep, goat, and cattle's respectively. Plant viral based innovative vaccines have been emerged ineffective vaccine development. This research describes the engineering and development of a new vaccine candidate by display immunogenic peptide using the carrier capsid protein of Papaya Mosaic Virus. The Capripox virus P32 immunogenic protein is homologous of the vaccinia virus H3L gene displayed PapMV CP. The antigenicity of P32 protein epitope lowest score among epitopes C-terminally docked epitopes are EP6 $>$ EP3 $>$ EP8 as well the lowest score among epitopes N-terminally docked epitopes are EP8 $>$ EP3 $>$ EP6 presented on the N-terminus of PMV CP region which are found to be suitable for epitope display. And these modelled immunogenic peptide could be used to develop a viral like particles. Epitope based Antibody developed against immunogenic epitopic regions can contribute to a novel and robust protection from infection. As well might be used for developing cost effective detection kits for Transboundary animal disease viruses.
\end{abstract}

Keywords Viral-like particles · Capripox viruses · Papaya mosaic viral capsid protein · B-Cell epitopes

\section{Abbreviations}

VLPS Viral-like particles

PMV CP Papaya mosaic virus capsid protein

LSDV Lymphy skin-disease virus

SPPV Sheeppox virus

K. M. Kumar

kirankumarmbbt@gmail.com

1 Department of Biotechnology, REVA University, Bengaluru 560064, India

2 National Institute of Veterinary Epidemiology and Disease Informatics, Bengaluru, India

3 Dayananda Sagar College of Engineering, Bengaluru, India

4 Department of Animal Behavior and Physiology, School of Biological Sciences, Madurai Kamaraj University, Madurai 625021, India
GPPV Goatpox virus

EPs Epitopes

\section{Introduction}

Virus-like particles have a multiple epitopes display on the surface comprises from viral antigens and have an ability self-assembled arranges the indigenous construction of viruses and they are lack of their viral genomes. These virallike particles can be formed from nucleocapsid or envelope proteins alone (Pushko et al. 2013). Based on high advantages of viral-like particles in safety, manufacturing and immunogenicity emerged as a foremost vaccine platform (Chen and Lai 2013). In biomedical applications of viral like particles shown more effective and safety a novel subunit 
vaccine, gene therapy vectors and diagnostic kits constructions. Plant viruses are emerging as promising vectors for use in innovative vaccination and development of diagnostic kits. Formation and production of viral like particles of immunogenic recombinant proteins in numerous plant systems have been deeply investigated and improved. In Vaccinology plant derived microbicides, vaccines and antibodies shown great attention and implemented. For the development of safety and effective mucosal and orally delivered plant created or produced viral like particles need a nominal process of the plant tissue (Santi et al. 2006). To develop a viral like particles and viral-based vectors, Papaya Mosaic Virus based viral nanoparticles are the competent vaccine platform. The immunogenic protein like epitopes or peptide antigens are fused with the capsid protein of mosaic virus at $\mathrm{N}$-terminal side and $\mathrm{C}$-terminal side of resulting in nanoparticles presenting surface-displays the epitopes. The fusion between immunogenic peptides and coat protein shows the stabilize the epitope towards carrying and enhances immunogenicity (Babin et al. 2013).

In the Poxviridae family, Capripoxvirus are large dsDNA, and enveloped viruses characterized by clinical symptoms like fever, papules, vesicles (rarely), internal lesions and death. These diseases are economically important and most serious infectious viral diseases of animal poxviruses which cause diseases in cattle, goat, and sheep and by a lumpy skin disease viral infection (LSDV), Goatpox virus (GPPV) and sheeppox virus (SHPPV) (Bhanuprakash et al. 2006; Tarello and Kinne 2007; Mirzaie et al. 2015). These Capripoxviruses based on their infectious and economic importance listed in group A diseases in the Office International disease Epizooties (OIE) (Varshovi et al. 2017). Capripoxviruses have the threats to become potential emerging disease because of global climatic change and due to porous international boundaries which facilitate for immigration of animals and their animal products from neighboring countries. These diseases are endemic in Africa, Middle East, Europe and Asia. The disease is transmitted through the infected animal direct contact or by contaminated objects (Tuppurainen et al. 2015). In cattle's LSD viral infection is a serious significant Transboundary, emerging skin viral disease and it is recognized and included in the OIE list (Mhemid and Hassan 2015). The capripox viral (sheep and goat pox virus) immunogenic p32 envelop protein is the homologous of P35 encoded by vaccinia viral H3L gene and locate membrane surface of the viral particles (Zhou et al. 2012).

Plant viruses are acting as emerging vectors to use and develop a novel vaccination strategies (Nuzzaci et al. 2006). VLC based Vaccines Engerix ${ }^{\circledR}$ and Recombivax ${ }^{\circledR}$ for hepatitis B and Gardasil ${ }^{\circledR}$ and Cervarix ${ }^{\circledR}$ for human papillomavirus commercially licensed (Crisci et al. 2013). Available vaccines against capripox viruses shows cross-protection and failure to provoke long-term protection against the various serovars of the pathogenic Capripox virus mixed strains.

The main aim is to predicit and map the B-cell epitopes (BCEp) on P32 in order to find a novel epitope based vaccine candidate. Expression of capsid proteins in microbial systems and their assembly into VLPs facilitate mass production of CP in vitro (Rostami et al. 2014). Therefore, the intend of this work was to modele and display of possible antigenic P32 BCEp on the surface of PapMV CP.

\section{Materials and Methods}

\section{Retrieval of Sequence and Multiple Sequence Alignments}

Capripox virus-P32 envelop protein is the homolog of P35 protein encoded H3L gene of vaccinia virus. The amino acid sequences was accessed from UniProtKB and National Centre for Biotechnology Information center (NCBI) (www.ncbi.nlm.nih.gov). As well Papaya Mosaic Virus Coat Protein (PMV CP) was also downloaded to predict the tertiary structure. The UniProt ID for Coat Protein on Papaya Mosaic Virus was P16596; and the immunogenic p32 accession numbers are AYI57795.1 is the major envelope protein Sheeppox virus, AAQ88213.1 envelope protein of Goat pox virus and AAD31773.1 P32 antigen of LSDV from these three Capripox viral p32 immunogenic protein carried out the NCBI protein BLAST (Altschul et al. 1997, 2005) multiple sequence alignment to understand structural similarity. As well, we carried out MSA of different sheep pox viral immunogenic $\mathrm{p} 32$ protein. The sequence numbers are AHB72723, AHB72720, AHI18587, ACR20671, AYO90717, AYO90726, AYO90724, AYO90723, AYO90722, AYO90721, AYI57795. The physico-chemical characterization of Capripox viral major envelop protein and papaya mosaic viral coat proteins was carried out by using Expasy's ProtParam server (https://us.expasy.org/tools/protp aram.html).

\section{Homology Modeling and Structural Validation}

Homology modelling and 3D structural prediction of PMV $\mathrm{CP}$ and Capripox vial envelop p32 proteins was carried out by using phyre2, (https://www.sbg.bio.ic.ac.uk/phyre2) (Kelley et al. 2015) and shows the advanced remote homology recognition method to construct 3D models. The evaluation of protein structural models was carriedout by using PROCHECK, Swiss-PdbViewer software and finally chosen model were 3D visualization was done using PyMOL (Guex and Peitsch 1997). 


\section{In Silico Epitope Mapping}

The main aim epitope prediction and mapping to identify and predict the binding sites of antigen-antibody interactions to enhance immuneresponce as well improvement of effective diagnostics, vaccines and immunotherapeutic compounds. The sequential, conformational immunogenic epitope of Capripox viral p32 were mapped from its predicted model using IEDB ElliPro: Antibody Epitope Prediction server (https://tools.immuneepitope.org/). The predicted epitopes were further analyzed prapable antigen or non-antigen by VaxiJen server with criteria of 0.3 threshold values and virus as the target organism. Based on the principle an ACC (Auto cross covariance) predicts defensive antigens renovation of protein sequence into identical vectors of principle amino acid. The predicted and selected epitope were modelled by PEP-FOLD server.3 (Idris et al. 2018).

\section{Coat Protein-Epitope Docking}

The HADDOCK is a high ambiguous interaction restraints online server used for the prediction of protein-protein flexible docking approach to model a biomolecular complexes. Based on highest low intermolecular energy cluster and representative docked structures was chosen analyzed for inter-molecular interactions. P32 epitopes were docked at the $\mathrm{C}$ and $\mathrm{N}$ terminal of the PMV CP using HADDOCK 2.2 webserver. (https://milou.science.uu.nl/services/HADDO CK2.2/haddock.php) (Ikram et al. 2018; Bhavaniprasad et al. 2013). The active and passive residues- required for protein docking studies- in PMV CP and P32 were predicted by, SPPIDER which were integrated in a single platform by CPORT. The epitopes were docked at the $\mathrm{N}$ and $\mathrm{C}$ terminal of PMV CP, with the corresponding active residues chosen at the instance. The PMV CP-Epitope docked complex with the lowest docked score was chosen at each termini for further analysis (Porollo and Meller 2007). Moming was Mapped B-cell epitopes and expressed N-terminal region and C-terminal region of NP of hemorrhagic fever virus (Moming et al. 2018) to understanding the structure and functions efficiently towards multi-epitope detection antigen as well epitope based vaccine.

\section{Results}

\section{Physicochemical Properties and Multiple Sequence Alignments}

The physicochemical structural properties of three envelop p32 proteins of capripox virus and PapMV coat protein was done. The parameters included the pI values, amino acid composition, coefficient, atomic composition, molecular weight aliphatic index, instability index and hydropathicity the detailed results were showed in Table 1.

The BLAST Results of capripoxvirus p32 envelop proteins were shown identies of Lumpy skin disease virus AAD31773.1 P32 antigen shown 315/323(98\%) Identities, Goatpox virus_AAQ88213.1 envelope protein P32 310/323(96\%) identies with sheeppox virus Sheeppox virus_AYI57795.1

Multiple sequence alignment of major envelop p32 protein selected sequences were shown variation at 26 position GPV virus Glycine (G) is present instead of Aspartic (D) acid where as in LSDV and SPV shown Aspartic acid. 46 amino acid position Asparagine (N) is similar both LSDV and SPV, SPV envelop protein shown extra Aspartic acid at 54 position where as in LSDV and GPV shown a gap or absent aminoacid at particular position. LSDV and GPV shown similar amino acid at 132,134 positions where as in

Table 1 Parameters computed using Expasy's ProtParam tool

\begin{tabular}{|c|c|c|c|c|}
\hline \multirow[t]{2}{*}{ Property } & \multicolumn{3}{|c|}{ Values of P32 major envelope protein } & \multirow[t]{2}{*}{ PMV CP } \\
\hline & Sheeppox virus & Goatpox virus & $\begin{array}{l}\text { Lumpy skin } \\
\text { disease virus }\end{array}$ & \\
\hline Number of amino acides & 323 & 322 & 322 & 215 \\
\hline Molecular weight & 37583.58 & 37474.57 & 37593.62 & 23033.09 \\
\hline Theoretical pl & 8.24 & 8.84 & 8.24 & 7.73 \\
\hline Total number of negatively charged residues(Asp+Glu) & 38 & 36 & 38 & 15 \\
\hline $\begin{array}{l}\text { Total number of positively residues } \\
\text { (Arg+Lys) }\end{array}$ & 40 & 41 & 40 & 16 \\
\hline Extinction coefficient (1.330, assuming all pairs of Cys residues form cystines) & 49975 & 51465 & 49975 & 17085 \\
\hline Extinction coefficient* (1.326, assuming all Cys residues are reduced) & 49850 & 51340 & 49850 & 16960 \\
\hline Instability index & 28.63 & 26.49 & 28.18 & 46.37 \\
\hline Aliphatic index & 103.81 & 100.78 & 102.61 & 72.28 \\
\hline Grand average of hdropathicity & -0.041 & -0.042 & -0.03 & -0.148 \\
\hline
\end{tabular}


AHI18587/Jilin/MEP

AHB72720/GanSUGT/11/2012/China AYI57795/NIVEDI/Jamakandi/2017 AYo90717/36/16/Tunis AYo90726/1517/12F/Tunisia AY090724/3337/10

AYO90723/TN

AYO96722/TN

AYO90721/TNO9/16

ACR20671/Pune/08

AHB72723/GanSuHN/12/2012/China

AHI18587/Jilin/MEP

AHB72720/GanSuGT/11/2012/China AYI57795/NIVEDI/Jamakandi/2017 AY090717/36/16/Tunis AYo90726/1517/12F/Tunisia AYO90724/3337/10

AYO90723/TN

AYO90722/TN

AYO90721/TN09/16

ACR20671/Pune/08

AHB72723/GanSuHN/12/2012/China

AHI18587/Jilin/MEP

AHB72720/GanSuGT/11/2012/China AYI57795/NIVEDI/Jamakandi/2017 AY090717/36/16/Tunis AY096726/1517/12F/Tunisia

AYO90724/3337/10

AYO90723/TN

AYO90722/TN

AYO90721/TNO9/16

ACR20671/Pune/08

AHB72723/GanSuHN/12/2012/China

AHI18587/Jilin/MEP

AHB72720/GanSuGT/11/2012/China AYI57795/NIVEDI/Jamakandi/2017 AY090717/36/16/Tunis AYo90726/1517/12F/Tunisia

AYO90724/3337/10

AY090723/TN

AYO90722/TN

AY090721/TN09/16

ACR20671/Pune/08

AHB72723/GanSuHN/12/2012/China

AHI18587/Jilin/MEP

AHB72720/GanSuGT/11/2012/China AYI57795/NIVEDI/Jamakandi/2017 AYO90717/36/16/Tunis AY090726/1517/12F/Tunisia AYO90724/3337/10

AYO90723/TN

AYO90722/TN

AY090721/TN09/16

ACR20671/Pune/08

AHB72723/GanSuHN/12/2012/China

AHI18587/Jilin/MEP

AHB72720/GanSuGT/11/2012/China AYI57795/NIVEDI/Jamakandi/2017 AYoge717/36/16/Tunis

AYo90726/1517/12F/Tunisia

AYO9日724/3337/10

AYO90723/TN

AYO90722/TN

AYO90721/TNO9/16

ACR20671/Pune/08

AHB72723/GanSuHN/12/2012/China
Epi-6

Epi-1

MADIPLYVIPIVGREIS PY PELKSDNDIFYKKVD VVDFKNSDVNFFLKDKKDDISLSY

MADIPLYVIPIVGREIS DV/PELKSDNDIFYKKVD VVKDFKNSDVNFFLKDKKDDISLSY

MADIPLYVIPIVGREIS PV/PELKSDNDIFYKKVD VKDFKNSDVNFFLKDKKDDISLSY

MADIPLYVIPIVGREIS PV/PELKSDNDIFYKKVD YVKDFKNSDVNFFLKDKKDDISLSY

MADIPLYVI IVGREIS WV/PELKSDNDIFYKKVD VVKDFKNSDVNFFLKDKKDDISLSY

MADIPLYVI IVGREIS PV/PELKSDNDIFYKKVD VKKDFKNSDVNFFLKDKKDDISLSY

MADIPLYVIPIVGREIS DV/PELKSDNDIFYKKVD TVKDFKNSDVNFFLKDKKDDISLSY

MADIPLYVIPIVGREISPYVPELKSDNDIFYKKVD VVKDFKNSDVNFFLKDKKDDISLSY

MADIPLYVI IVGREIS PV/PELKSDNDIFYKKVD VKKDFKNSDVNFFLKDKKDDISLSY

MADIPLYVIPIVGREIS DV/PELKSDNDIFYKKVD VVDFKNSDVNFFLKDKKDDISLSY

MADIPLYVI IVGREIS UYPELKSDNDIFYKKVD VVDFKNSDVNFFLKDKKDDISLSY

Epi-2

Epi-3

KLLIWE VEKSGGVENFTEYFSGLCNALCTKEA SSIAKHFSLWKSYAD IDIKNSEN FI

KLLIWE VVESGGVENFTEYFSGLCNALCTKEA SSIAKHFSLWKSYADIDIKNSEN FI

KLLIWE VVEKSGGVENFTEYFSGLCNALCTKEA. SSIAKHFSLWKSYADADIKNSEN F FI

KLLIWE VVEKSGGVENFTEYFSGLCNALCTKEA SSIAKHFSLWKSYADIDIKNSEN FI

KLLIWE VVEKSGGVENFTEYFSGLCNALCTKEA. SSIAKHFSLWKSYAD ADIKNSEN FI

KLLIWE VVEKSGGVENFTEYFSGLCNALCTKEA. SSIAKHFSLWKSYADADIKNSEN FFI

KLLIWE VVEKSGGVENFTEYFSGLCNALCTKEA SSIAKHFSLWKSYADLDIKNSEN FI

KLLIWE VVEKSGGVENFTEYFSGLCNALCTKEA. SSIAKHFSLWKSYADADIKNSEN FI

KLLIWE VVEKSGGVENFTEYFSGLCNALCTKEA. SSIAKHFSLWKSYADADIKNSEN F FI

KLLIWE VVEKSGGVENFTEYFSGLCNALCTKEAN SSIAKHFSLWKSYADWDIKNSEN FI

KLLIWE VVEKSGGVENFTEYFSGLCNALCTKEA. SSIAKHFSLWKSYADADIKNSEN FI

******

Epi-8

Epi-4

WIEDDNTLKDSVIIHNI IIEIMEKN DDIFQLR \&TFHNSNSRILFNQENNNFMYSYTGGY

WIEDDNTLKDSIIIHNIIIEMQEKN DIFQLR QTFHNSNSRILFNOENNNFMYSYTGGY

WI EDDNTLKDSI IIHNI IIEMOE KN FDIFOLR ETFHNSNSR I LFNOENNNFMYSYTGGY

WIEDDNTLKDSIIIHNI IEMQEKN DIFQLR Q TFHNSNSRILFNQENNNFMYSYTGGY

WI EDDNTLKDSI IIHNI I EMOEKN DOIFOLR RTFHNSNSRILFNOENNNFMYSYTGGY

WIEDDNTLKDSI IIHNI IIEMOEKN FDIFOL R TTFHNSNSRILFNOENNNFMYSYTGG)

WIEDDNTLKDSI IIHNIIIEMQE KN DIFQLR TTFHNSNSRILFNQENNNFMYSYTGGY

WI EDDNTLKDSIIIHNIIIEMOEKN DIFQLR ETFHNSNSRILFNOENNNFMYSYTGGY

WIEDDNTLKOSIIIHNI IIEMOEKN FDIFOLR TTFHNSNSRILFNOENNNFMYSYTGG

WIEDDNTLKDSIIIHNI IIEMQEKN DIFQLR ETFHNSNSRILFNQENNNFMYSYTGGY WIEDDNTLKDSIIIHNIEIEMQEKN DIFQLR Q TFHNSNSRILFNQENNNFMYSYTGGY

Epi-7

Epi-5

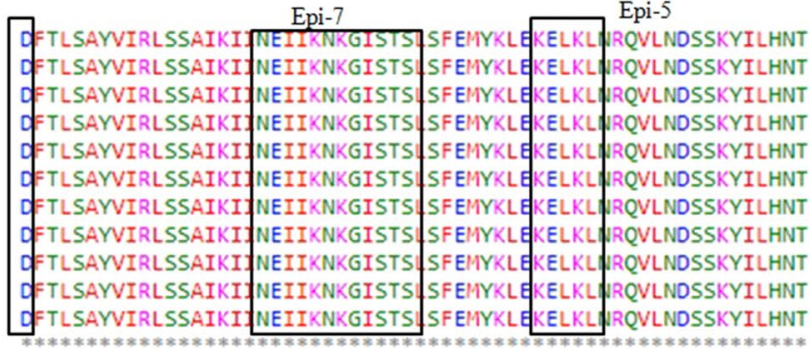

KYLSKKRANEMKNGIWNRVGKIVMAHRFPDFSYYVSHPLVSFFGIFDISIIGALIILFIII KYLSKKRANEMKNGIWNRVGKWHAHRFPDFSYYVSHPLVSFFGIFGISIIGALIILFIII KYLSKKRANEMKNGIWNRVGKIUAAHRFPDFSYMSHPLVSFFGIFDISIIGALIILFIII KYLSKKRANEMKNGIWNRVGKLWHAHRFPDFSYYVSHPLVSFFGIFDISIIGALIILFIII KYLSKKRANEMKNGIWNRVGKWWAHRFPDFSYVSHPLVSFFGIFDISIIGALIILFIII KYLSKKRANEMKNGIWNRVGKIVIAHRFPDFSYYVSHPLVSFFGIFDISIIGALIILFIII KYLSKKRANEMKNGIWNRVGKIUAAHRFPDFSYVSHPLVSFFGIFDISIIGALIILFIII KYLSKKRANEMKNGIWNRVGKWMAHRFPDFSYYSHPLVSFFGIFDISIIGALIILFIII KYLSKKRANEMKNGIWNRVGKWUAHRFPDFSYYVSHPLVSFFGIFDISIIGALIILFIII KYLSKKRANEMKNGIWNRVGKIMAAHRFPDFSYYSHPLVSFFGIFDISIIGALIILFIII KYLSKKRANEIMKNGIWNRVGKIVMAHRFPDFSYYVSHPLVSFFGIFDISIIGALIILFIII

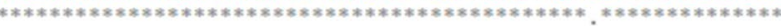

MIIFNLNSKLLWFLAGMLFTYII MIIFNLNSKLLWFLAGMLFTYIV MIIFNLNSKLLWFLAGMLFTYII MIIFNLNSKLLWFLAGMLFTYII MIIFNLNSKLLWF LAGMLFTYII MIIFNLNSKLLWFLAGMLFTYI MIIFNLNSKLLUFLAGMLFTYII MIIFNLNSKLLWF LAGMLFTYII MIIFNLNSKLLWFLAGMLFTYII MIIFNLNSKLLWFLAGMLFTYII MIIFNLNSKLLWF LAGMLFTYIV

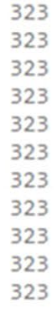


४Fig. 1 Multiple sequence alignment of P32: Multiple sequence alignment of P32 from 11 different strains of Capripox viruses were tested by T-Coffee program (https://tcoffee.crg.cat/apps/tcoffee/do:regular). Among all selected capripox viral p32 sequences colured regions in the above alignment showen perfect sequence alignment. And the predicted epitopes were marked as boxes in the particular regions

SPV at 132 positions Serine and 134 position Isoleucine variations was observed. at 290, 325 amino acid position SPV and LSDV were shown Isoleucine but GPV shown Methionine at 290 position and at 322 position Valine variation. SPV and GPV were shown similar amino acid at 305 position where as LSDV Aspartic acid (D) was observed. multiple sequence alignment of different sheeppox viral immunogenic p32 protein and their epitope predicted sequences were showened in Fig. 1.These results may help to analyse identification species basic envelop based diagnostic marker development.

\section{Homology Modelling and Structural Validation}

The immunogenic p32 protein of and papaya mosaic virus tertiary structure was predicted by PHYRE2, this server can perform ab initio modelling to improve models figure. The modelled structure was validated by Ramachandran plot generated using Procheck and RAMPAGE software (Lovell et al. 2002). The detailed protein structural prediction 3D modelled and validation of proteins results were shown below images. PMV CP predicted and modelled protein showed in Fig. 2 and P32 protein showed in Fig. 3.

\section{In Silico Epitope Mapping and Probable Antigen Validation}

The predicted epitope peptides length of P32 is these positions-EP1 (21-36), EP2 (66-94), EP3 (110-118), EP4 (155-181), EP5 (220-224), EP6 (11-19), EP7 (199-210), EP8 (140-147) (Fig. 2). Further analysis of these epitopes were verified on VaxiJen web server, where the antigenicity values were; EP1 (0.1976), EP2 (0.3483), EP3 (0.5925), EP4 (0.3671), EP5 (-), EP6 (0.7832), EP7 (0.2495) and EP8 (1.5581). All probable antigenic epitopes (EP3, EP6 and EP8) was taken for further analysis. The results were tabulated in Table 2.

\section{Coat Protein-Epitope Docking}

Selected probable antigenic epitopes (EP3, EP6 and EP8) were used to display on Papaya Mosaic Virus Coat protein. HADDOCK web server clustered the structures and the top ten were analyzed as they have the most reliable structural interaction based on the score obtained by each cluster. The epitopes when individually docked at $\mathrm{N}$ and C-terminal region of the PMVCP (Kumar et al. 2015). The docked (a) Structural model of PaMV CP protein

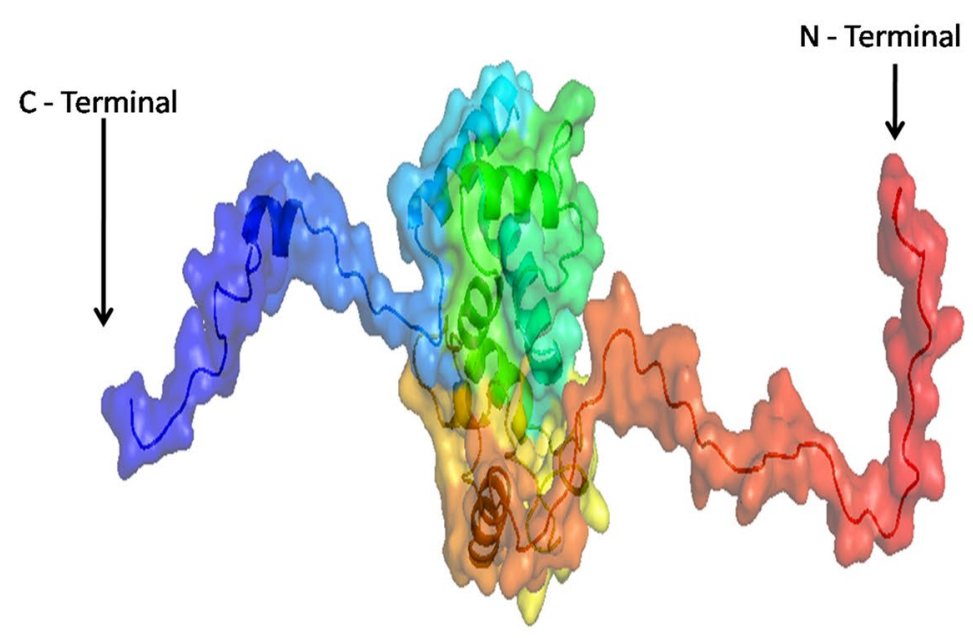

(b) Ramachandran plot PaMV CP

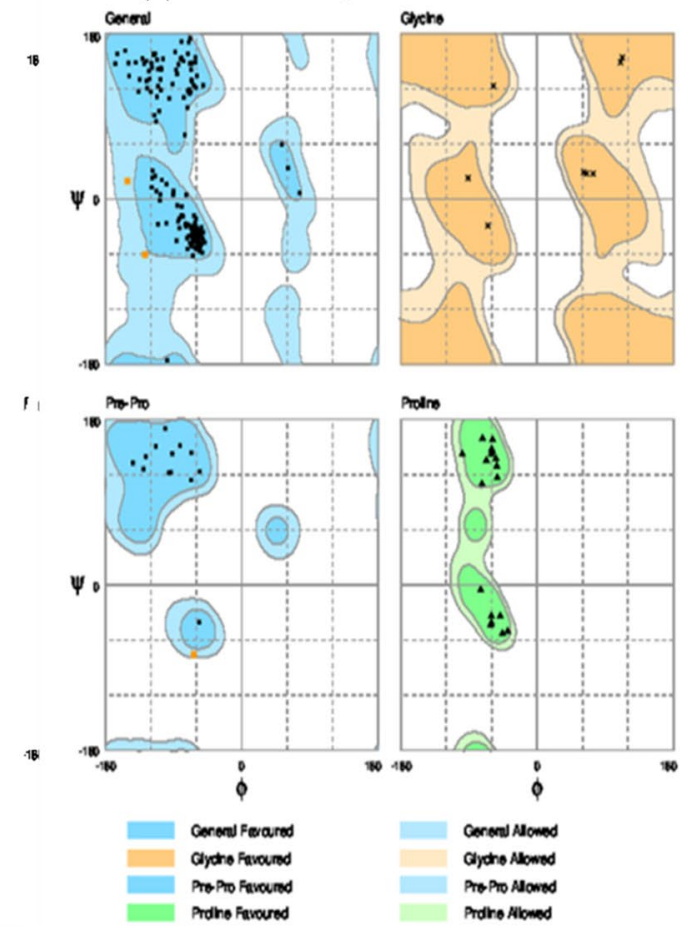

Fig. 2 Modelled and validated Papaya Mosaic Viral Coat Protein Pyre2 and RAMPAGE 


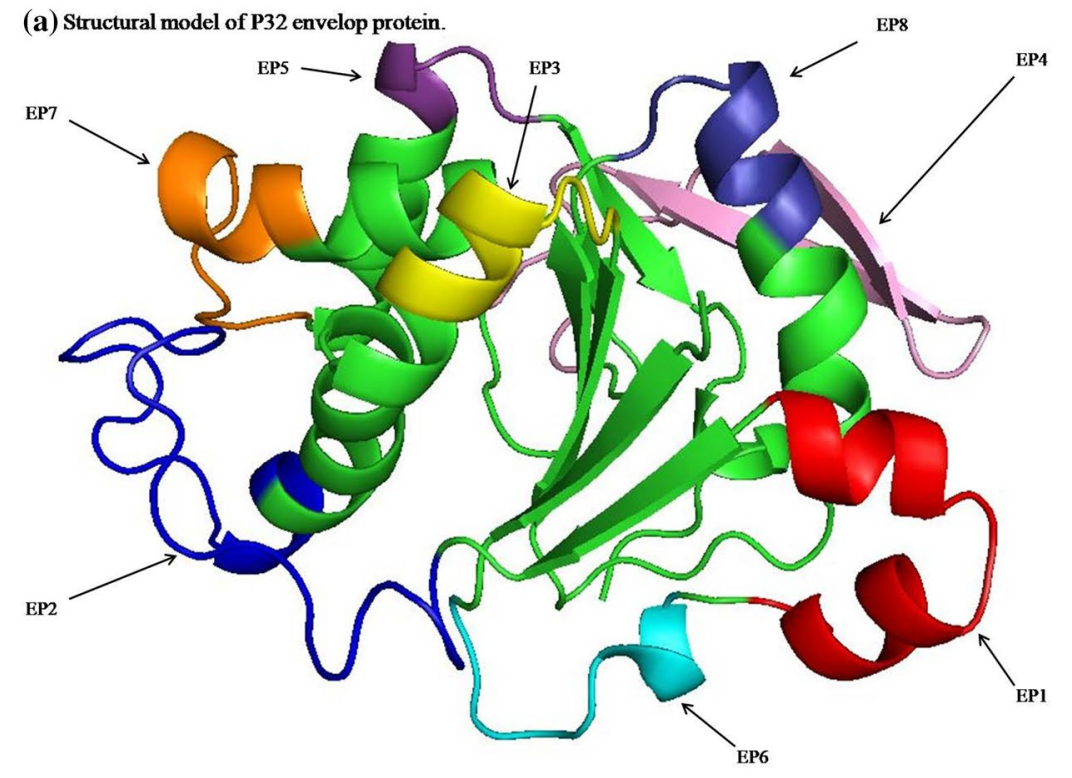

(b) Ramachandran plot

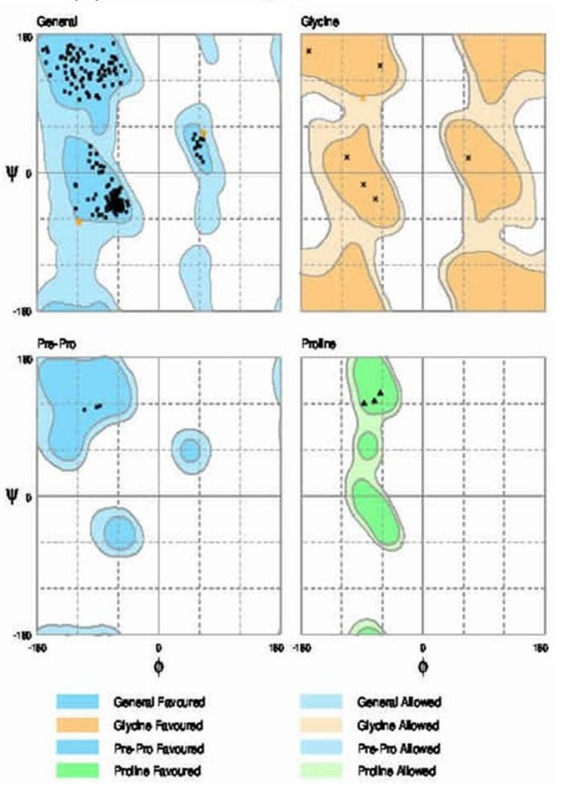

Fig. 3 Modelled and predicted structure of p32 protein and predicted different epitopic regions highlighted in different colours

Table 2 Prediction and verification of P32 epitopes by VaxiJen antigen prediction server

\begin{tabular}{|c|c|c|c|c|c|c|}
\hline Epitope Sl. No & Start & End & Peptide & $\begin{array}{l}\text { Number of } \\
\text { residues }\end{array}$ & Score & $\begin{array}{l}\text { VaxiJen prob- } \\
\text { able antigenic } \\
\text { score }\end{array}$ \\
\hline Ep1 & 21 & 36 & PELKSDNDIFYKKVDT & 16 & 0.789 & $0.1976(\mathrm{NP})$ \\
\hline Ep2 & 66 & 94 & EKVEKSGGVENFTEYFSGLCNALCTKEAK & 29 & 0.711 & $0.3483(\mathrm{NP})$ \\
\hline Ep3 & 110 & 118 & ADIKNSENK & 9 & 0.688 & 0.5925 (AP) \\
\hline Ep4 & 155 & 181 & TFHNSNSRILFNQENNNFMYSYTGGYD & 27 & 0.673 & $0.3671(\mathrm{NP})$ \\
\hline Ep5 & 220 & 224 & KELKL & 5 & 0.643 & - \\
\hline Ep6 & 11 & 19 & IVGREISDV & 9 & 0.613 & 0.7832 (AP) \\
\hline Ep7 & 199 & 210 & NEIIKNKGISTS & 12 & 0.614 & $0.2495(\mathrm{NP})$ \\
\hline Ep8 & 140 & 147 & IEMQEKNI & 8 & 0.526 & 1.5581 (AP) \\
\hline
\end{tabular}

$A$ probable antigen, $N A$ probable non-antigen, $N P$ non probable antigen, $A P$ probable antigen

results given scores based on Van der Waal's energy, electrostatic energy, desolvation energy, binding energy and buried surface area. Docking results were analyzed and tabulated in detail Table 3 (Dominguez et al. 2003; van Zundert et al. 2016).

EP6 had the lowest score among other epitopes terminally docked. At the $\mathrm{C}$ terminal of PMV CP, the score was $-62.0 \pm 5.2$ and at the $\mathrm{N}$ terminal it was $-55.4 \pm 2.0$. The lowest score among epitopes C-terminally docked is EP6 $>$ EP3 $>$ EP8 as well the lowest score among epitopes N-terminally docked is EP8 $>$ EP3 $>$ EP6 and the models are displayed in surface models using PyMOL showed in Fig. 4 and the Interaction between PMV CP at C and N-terminus regions with CEp3, CEp6 and CEp8 Epitopes was analysed by PDB SUM (Laskowski et al. 2018) showed in Figs. 5 and 6. Evaluation of predicted models by Rampage the results were tabulated in Table 4.

\section{Interaction Between PMV CP N and C-Terminus Regions with Epitopes}

\section{CEp3}

The interaction between the PaMV CP with epitope 3 at C-terminus was observed the number of hydrogen bonds 7 and number of non-bonded contacts 66. C-terminus protein interactive active amino acid residues LYS 198, GLN 200, SER 202, SER 204, SER 204, PRO 206, GLU 215 interacted 
Table 3 Predicted and assessment of docked complexes

\begin{tabular}{|c|c|c|c|c|c|c|c|c|}
\hline \multicolumn{2}{|c|}{ Models of PMV CP-epitope } & \multirow{2}{*}{$\begin{array}{l}\text { HADDOCK } \\
\text { score }\end{array}$} & \multirow[t]{2}{*}{ Z-score } & \multirow{2}{*}{$\begin{array}{l}\text { Van der Waals } \\
\text { energy (Kcal } \\
\left.\text { mol }^{-1}\right)\end{array}$} & \multirow{2}{*}{$\begin{array}{l}\text { Electrostatic } \\
\text { energy (Kcal } \\
\left.\mathrm{mol}^{-1}\right)\end{array}$} & \multirow{2}{*}{$\begin{array}{l}\text { Desolvation } \\
\text { energy (Kcal } \\
\left.\mathrm{mol}^{-1}\right)\end{array}$} & \multirow{2}{*}{$\begin{array}{l}\text { Restraints vio- } \\
\text { lation energy } \\
\left(\mathrm{Kcal} \mathrm{mol}^{-1}\right)\end{array}$} & \multirow{2}{*}{$\begin{array}{l}\text { Buried surface } \\
\text { area }\end{array}$} \\
\hline $\begin{array}{l}\text { Coat protein } \\
\text { terminal }\end{array}$ & Epitope name & & & & & & & \\
\hline \multirow{3}{*}{$\begin{array}{l}\text { Carboxy termi- } \\
\text { nal (C) }\end{array}$} & EP3 & $-55.6 \pm 8.2$ & -1.7 & $-29.6 \pm 1.9$ & $-135.0 \pm 49.1$ & $-8.4 \pm 4.0$ & $94.2 \pm 29.61$ & $954.8 \pm 38.8$ \\
\hline & EP6 & $-62.0 \pm 5.2$ & -1.4 & $-33.3 \pm 4.5$ & $-75.5 \pm 26.4$ & $-21.7 \pm 1.6$ & $81.8 \pm 42.08$ & $976.1 \pm 43.6$ \\
\hline & EP8 & $-52.4 \pm 2.4$ & -1.4 & $-24.9 \pm 8.9$ & $-117.8 \pm 25.4$ & $-13.7 \pm 3.3$ & $97.5 \pm 31.10$ & $909.0 \pm 72.0$ \\
\hline \multirow{3}{*}{$\begin{array}{l}\text { Amino termi- } \\
\operatorname{nal}(\mathrm{N})\end{array}$} & EP3 & $-56.7 \pm 4.8$ & -1.8 & $-32.9 \pm 4.3$ & $-102.7 \pm 30.7$ & $-13.2 \pm 9.6$ & $98.5 \pm 47.72$ & $953.1 \pm 84.4$ \\
\hline & EP6 & $-55.4 \pm 2.0$ & -1.6 & $-34.5 \pm 5.7$ & $-99.4 \pm 35.7$ & $-8.3 \pm 2.6$ & $72.9 \pm 35.11$ & $1027.6 \pm 78.3$ \\
\hline & EP8 & $-57.5 \pm 2.1$ & -1.9 & $-35.5 \pm 1.3$ & $-144.1 \pm 12.9$ & $-6.6 \pm 4.2$ & $135.0 \pm 34.00$ & $1024.5 \pm 61.6$ \\
\hline
\end{tabular}

with epitope 3 chain residues SER 6, ILE 3, LYS 4, LYS 4, LYS 4, ASN 8, LYS 9.

\section{CEp6}

The interaction between the PaMV CP with epitope 6 at C-terminus was observed the number of hydrogen bonds 8 and number of non-bonded contacts 73. C-terminus protein interactive active amino acid residues LYS 198, GLY 199,
GLN 200, ILE 201, SER 204, PRO 206, THR 207, GLU 215 interacted with epitope 6 chain residues SER 6, ILE 3, LYS 4, LYS 4, LYS 4, ASN 8, LYS 9.

\section{CEp8}

The interaction between the PaMV CP with epitope 8 at C-terminus was observed the number of hydrogen bonds 8

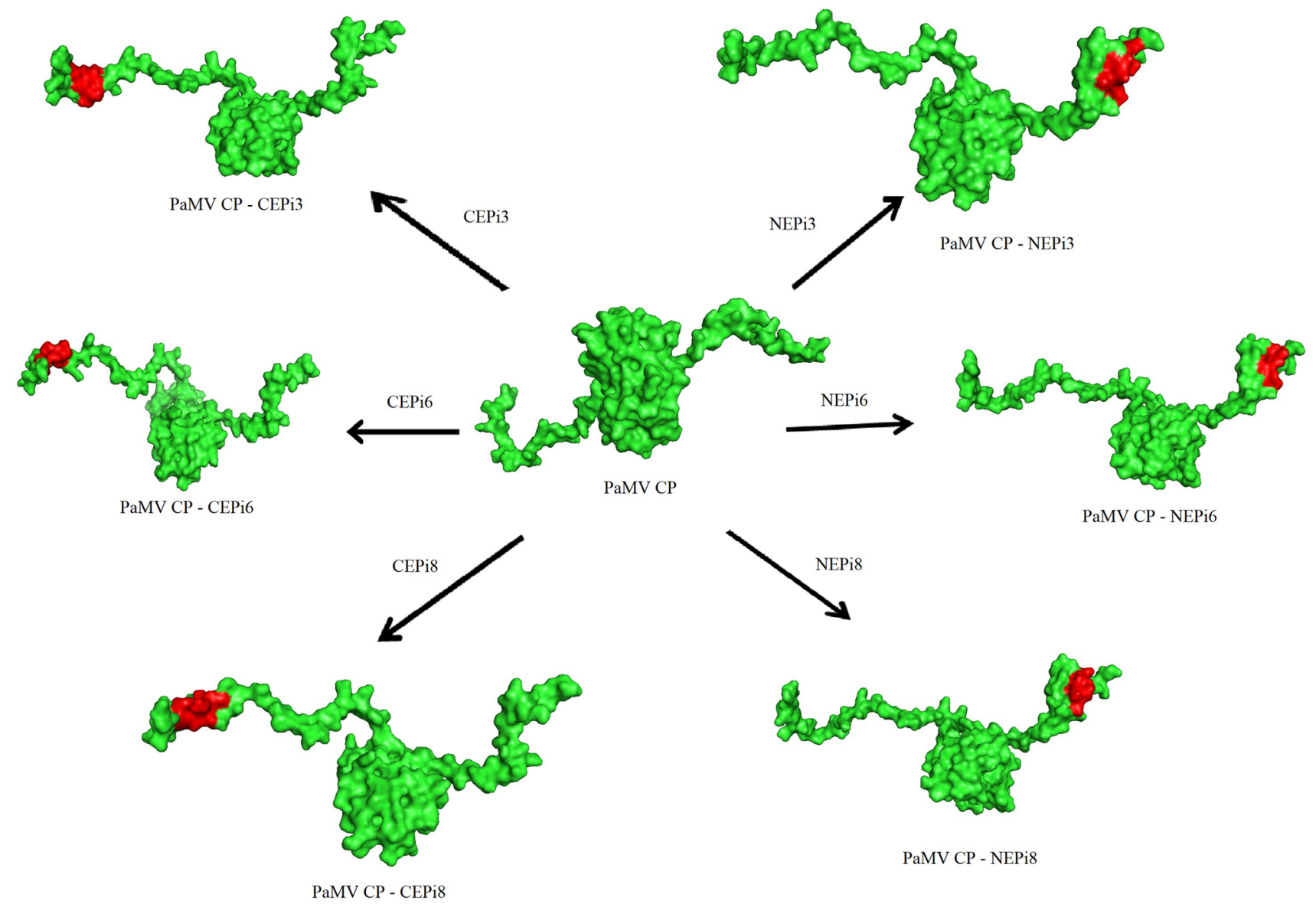

Fig. 4 PMV CP- immunogenic Capripox viral envelop P32 protein epitopes docking at N, C termini: Protein-epitope docking of P32 epitopes at $\mathrm{N}, \mathrm{C}$ by using HADDOCK server and the models are displayed in surface models using PyMOL 


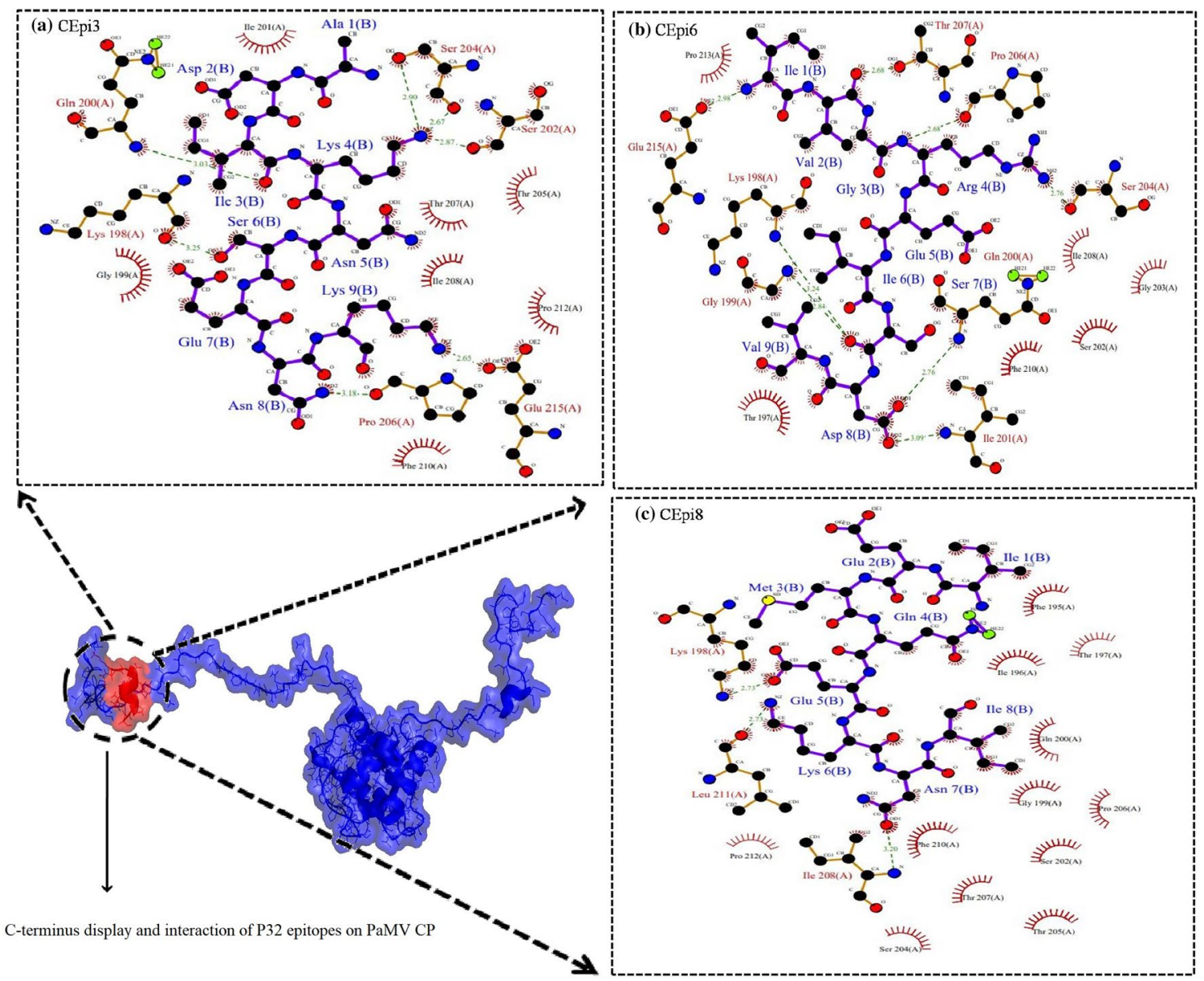

Fig. 5 Interaction between PMV CP at C-terminus regions with CEp3, CEp6 and CEp8 epitopes

and number of non-bonded contacts 62 . C-terminus protein interactive active amino acid residues LYS 198, ILE 208, LEU 21 linteracted with epitope 8 chain residues GLU 5, ASN 7, LYS 6.

\section{NEp3}

The interaction between the PaMV CP with epitope 3 at $\mathrm{N}$-terminus was observed the number of hydrogen bonds 8 and number of non-bonded contacts 95 . N-terminus protein interactive active amino acid residues LYS 3, THR 8, THR 8, ASN 10, ASN 10, ILE 16, ILE 16, GLN 18 interacted with epitope 3 chain residues LYS 9, ASP 2, ASN 5, SER 6, ASN 8, ASP 2, ILE 3, LYS 4.

\section{NEp6}

The interaction between the PaMV CP with epitope 6 at $\mathrm{N}$-terminus was observed the number of hydrogen bonds 4 and number of non-bonded contacts 84 . N-terminus protein interactive active amino acid residues MET 6, PRO 9, ALA 12, GLN 18 interacted with epitope 6 chain residues GLY 3, ARG 4, ARG 4, ASP 8.

\section{NEp8}

The interaction between the PaMV CP with epitope 8 at $\mathrm{N}$-terminus was observed the number of hydrogen bonds 8 and number of non-bonded contacts 99 . N-terminus protein interactive active amino acid residues SER 5, SER 7, ALA 15, ILE 16, GLN 18, GLN 18, GLU 19, MET 21 interacted with epitope 8 chain residues GLN 4, GLU 5, GLU 2, ILE 1 (Tables 5 and 6).

\section{Discussion}

Capripoxviruses are the genus comprises sheeppox, goatpox and Lymphyskin disease viruses in the poxviridae family, based on economic importance and infectious 


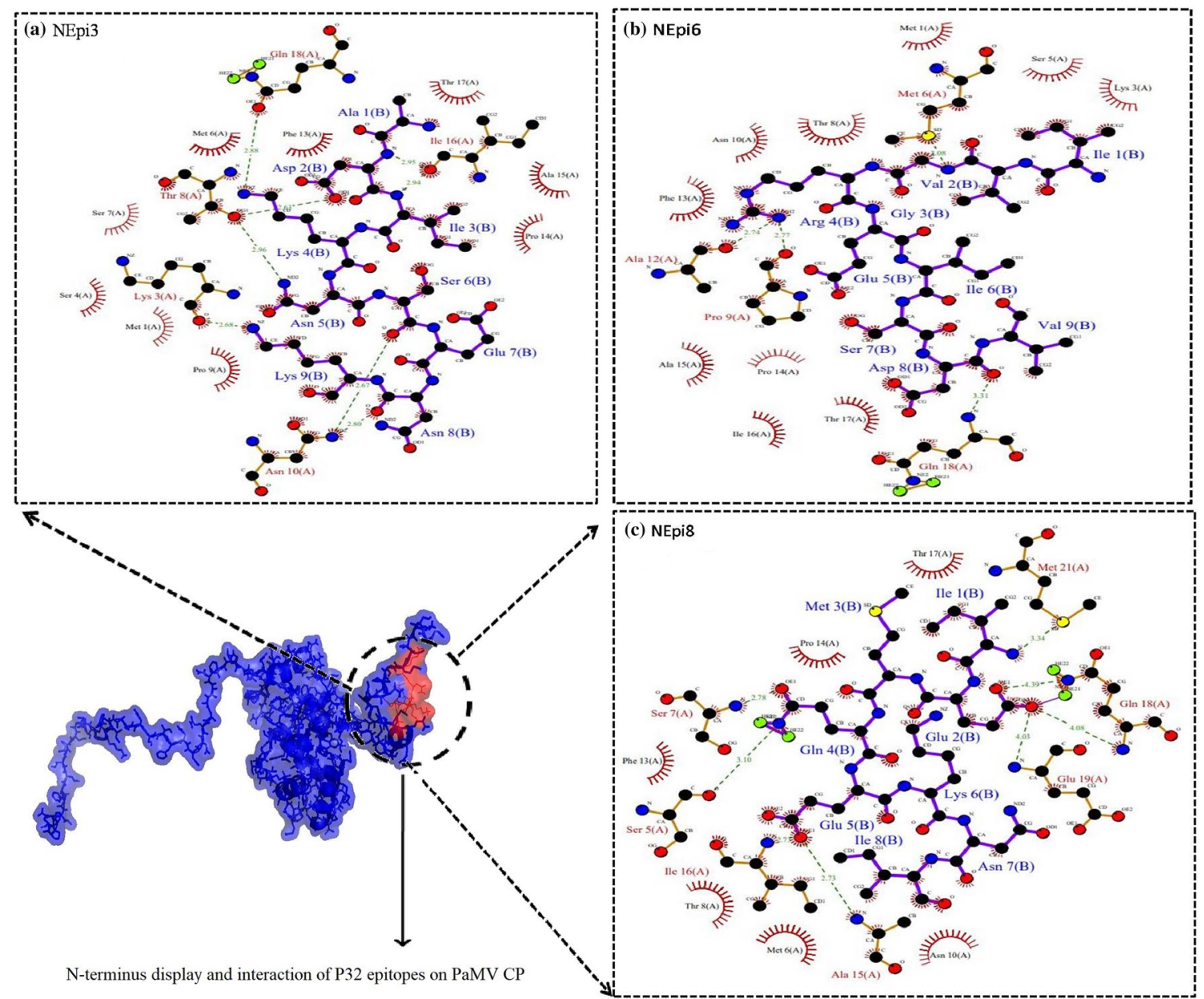

Fig. 6 Interaction between PMV CP at N-terminus regions with NEp3, NEp6 and NEp8 epitopes

Table 4 Evaluation of predicted models by Rampage

\begin{tabular}{|c|c|c|c|c|}
\hline \multicolumn{2}{|c|}{ Models of PMV CP-epitope } & \multirow{2}{*}{$\begin{array}{l}\text { Number of residues } \\
\text { in favoured region }\end{array}$} & \multirow{2}{*}{$\begin{array}{l}\text { Number of residues } \\
\text { in allowed region }\end{array}$} & \multirow{2}{*}{$\begin{array}{l}\text { Number of } \\
\text { residues in outlier } \\
\text { region }\end{array}$} \\
\hline Coat protein terminal & Epitope name & & & \\
\hline P32 & - & $210(98.6 \%)$ & $3(1.4 \%)$ & $0(0.0 \%)$ \\
\hline PMV & - & $210(98.6 \%)$ & $3(1.4 \%)$ & $0(0.0 \%)$ \\
\hline \multirow[t]{3}{*}{ Carboxy terminal (C) } & EP3 & $204(92.7 \%)$ & $14(6.4 \%)$ & $2(0.9 \%)$ \\
\hline & EP6 & $203(92.3 \%)$ & $14(6.4 \%)$ & $3(1.4 \%)$ \\
\hline & EP8 & $206(94.1 \%)$ & $11(5.0 \%)$ & $2(0.9 \%)$ \\
\hline \multirow[t]{3}{*}{ Amino terminal $(\mathrm{N})$} & EP3 & $209(95.0 \%)$ & $10(4.5 \%)$ & $1(0.5 \%)$ \\
\hline & EP6 & $198(90.0 \%)$ & $20(9.1 \%)$ & $2(0.9 \%)$ \\
\hline & EP8 & $208(95.0 \%)$ & $10(4.6 \%)$ & $1(0.5 \%)$ \\
\hline
\end{tabular}

nature these viruses are considered as the transboundary and OIE notifiable diseases of the developing the world and is always the threat of spreading of Capripox viruses into new geographic regions. The diseases show economic impact directly by causing morbidity and mortality as well by reducing reproduction performance, quality of wool and meat poisoning on international trade. Hence there is a need to identify Capripox virus isolates effects for both sheep and goats, leads may be able to develop single 
Table 5 Interaction between PMV CP at C-terminus regions with CEp3, CEp6 and CEp8 epitopes

\begin{tabular}{|c|c|c|c|c|c|c|c|c|c|c|c|c|}
\hline \multicolumn{6}{|c|}{ Protein chain A T O M 1} & & \multicolumn{6}{|c|}{ Epitope chain A T O M 2} \\
\hline CEp3 & Atom no & Atom name & Res name & Res no & Chain & & Atom no & Atom name & Res name & Res no & Chain & Distance \\
\hline 1 & 1830 & $\mathrm{O}$ & LYS & 198 & A & $<-$ & 2028 & OG & SER & 6 & B & 3.25 \\
\hline 2 & 1836 & $\mathrm{~N}$ & GLN & 200 & A & $->$ & 1999 & $\mathrm{O}$ & ILE & 3 & B & 3.03 \\
\hline 3 & 1864 & $\mathrm{O}$ & SER & 202 & A & $<-$ & 2007 & $\mathrm{NZ}$ & LYS & 4 & B & 2.87 \\
\hline 4 & 1877 & $\mathrm{O}$ & SER & 204 & A & $<-$ & 2007 & $\mathrm{NZ}$ & LYS & 4 & B & 2.67 \\
\hline 5 & 1874 & OG & SER & 204 & A & $<-$ & 2007 & $\mathrm{NZ}$ & LYS & 4 & B & 2.9 \\
\hline 6 & 1893 & $\mathrm{O}$ & PRO & 206 & A & $<-$ & 2048 & ND2 & ASN & 8 & B & 3.18 \\
\hline 7 & 1972 & OE1 & GLU & 215 & A & $<-$ & 2060 & $\mathrm{NZ}$ & LYS & 9 & B & 2.65 \\
\hline CEp6 & Atom no & Atom name & Res name & Res no & Chain & & Atom no & Atom name & Res name & Res no & Chain & Distance \\
\hline 1 & 1818 & $\mathrm{~N}$ & LYS & 198 & A & $->$ & 2041 & $\mathrm{O}$ & SER & 7 & B & 3.24 \\
\hline 2 & 1831 & $\mathrm{~N}$ & GLY & 199 & A & $->$ & 2041 & $\mathrm{O}$ & SER & 7 & B & 2.84 \\
\hline 3 & 1836 & $\mathrm{~N}$ & GLN & 200 & A & $->$ & 2047 & OD1 & ASP & 8 & B & 2.76 \\
\hline 4 & 1848 & $\mathrm{~N}$ & ILE & 201 & A & $->$ & 2048 & OD2 & ASP & 8 & B & 3.09 \\
\hline 5 & 1877 & $\mathrm{O}$ & SER & 204 & A & $<-$ & 2010 & $\mathrm{NH} 2$ & ARG & 4 & B & 2.76 \\
\hline 6 & 1893 & $\mathrm{O}$ & PRO & 206 & A & $<-$ & 1998 & $\mathrm{~N}$ & ARG & 4 & B & 2.68 \\
\hline 7 & 1898 & OG1 & THR & 207 & A & $->$ & 1992 & $\mathrm{O}$ & VAL & 2 & B & 2.68 \\
\hline 8 & 1973 & OE2 & GLU & 215 & A & $<-$ & 1976 & $\mathrm{~N}$ & ILE & 1 & B & 2.98 \\
\hline CEp8 & Atom no & Atom name & Res name & Res no & Chain & & Atom no & Atom name & Res name & Res no & Chain & Distance \\
\hline 1 & 1825 & $\mathrm{NZ}$ & LYS & 198 & A & $->$ & 2023 & OE2 & GLU & 5 & B & 2.73 \\
\hline 2 & 1903 & $\mathrm{~N}$ & ILE & 208 & A & $->$ & 2044 & OD1 & ASN & 7 & B & 3.2 \\
\hline 3 & 1944 & $\mathrm{O}$ & LEU & 211 & A & $<-$ & 2033 & $\mathrm{NZ}$ & LYS & 6 & B & 2.73 \\
\hline
\end{tabular}

immune targeting single vaccine to protect the species (Burranboina et al. 2018; Madhavan et al. 2016).

In this research firstly we carried out conservancy of immunogenic envelop p32 protein between different species of SPPV, GPV and LSDV. As well-screened 11 sheeppox viral conservancy of $\mathrm{p} 32$ protein epitopes was carried out towards the study of universal epitopes based vaccine development requires conserved amino acids of a protein among the various pathogenic strains. Multiple amino acid sequence alignment of $\mathrm{p} 32$ for 11 different strains of sheeppox virus NIVEDI/Jamakandi/2017 (AYI57795), GanSuHN/12/2012/China (AHB72723), GanSuGT/11/2012/China (AHB72720), Jilin (AHI18587), 36/16 Tunis (AYO90717), 1517/12F Tunisia (AYO90726), 3337/10 (AYO90724), TN 13/15 (AYO90723), TN 10/16 (AYO90722), TN09/16 (AYO90721), Pune-08 (ACR20671), Were analyzed by using the T-Coffee program Fig. 1.

Viral like particles are the multiprotein structures mimicking viable native viruses, in immunogenic antigen structure but lack of the viral genomic material subsequently they are non-replicative with non-infectious nature as well they retain their capability to penetrate cells and shows the immense applications like they offer a safe substitute to inactivated and attenuated viral vaccine enhancement (Naskalska and Pyrc 2015). These VLPs are the appearance to the native form virus purified from infected source plants as well noninfectious. VLPs are being used by the polyvalent foreign epitope peptide display on their viral capsid protein surface to improve their presentation, immunogenicity as well host immune reaction (Kumar et al. 2009). Plant viral capsid proteins put forward the benefit of uniformity with respect to size, shape and have capability to self assembling into highly repeating nano- VLPs structures. Plant-based VLPS have a capable to exhibit tolerance to against $\mathrm{pH}$ and high temperature, structurally particular chemical attachment sites and resist intracellular environment like resist towards proteases and nucleases as well as these VLPs have a cargo capacity like they may suitable intended for transporting drugs to the affected area. Due to nonpathogenic nature of plant based viral nanoparticles may ideal selection for therapeutic applications. These structures are lack of viral genomic material, hence they are non-replicative or causing any type of infection (Shoeb and Hefferon 2019).

The immunogenic peptide display on a suitable platform based on evaluating the binding capability of epitope peptide-like display on surface of phage, bacteria, insect, yeast cells or mammalian cells (Potocnakova et al. 2016). Babin et al. 2013 was studied the fusion of the epitope stabilizes and it improves the immunogenicity. The PMV vaccine platform have a ability to provoke a enduring memory response 
Table 6 Interaction between PMV CP at N - Terminus regions with NEp3, NEp6 and NEp8 Epitopes

\begin{tabular}{|c|c|c|c|c|c|c|c|c|c|c|c|c|}
\hline \multicolumn{6}{|c|}{ Protein chain A T O M 1} & & \multicolumn{6}{|c|}{ Epitope chain A T O M 2} \\
\hline NEp3 & Atom no & Atom name & Res name & Res no & Chain & & Atom no & Atom name & Res name & Res no & Chain & Distance \\
\hline 1 & 30 & $\mathrm{O}$ & LYS & 3 & A & $<-$ & 2060 & $\mathrm{NZ}$ & LYS & 9 & $\mathrm{~B}$ & 2.68 \\
\hline 2 & 68 & OG1 & THR & 8 & A & $->$ & 1987 & OD1 & ASP & 2 & B & 2.63 \\
\hline 3 & 68 & OG1 & THR & 8 & A & $<-$ & 2019 & ND2 & ASN & 5 & B & 2.96 \\
\hline 4 & 86 & ND2 & ASN & 10 & A & $->$ & 2031 & $\mathrm{O}$ & SER & 6 & $\mathrm{~B}$ & 2.67 \\
\hline 5 & 86 & ND2 & ASN & 10 & $\mathrm{~A}$ & $->$ & 2052 & $\mathrm{O}$ & ASN & 8 & B & 2.8 \\
\hline 6 & 139 & $\mathrm{O}$ & ILE & 16 & A & $<-$ & 1982 & $\mathrm{~N}$ & ASP & 2 & B & 2.95 \\
\hline 7 & 139 & $\mathrm{O}$ & ILE & 16 & A & $<-$ & 1991 & $\mathrm{~N}$ & ILE & 3 & B & 2.94 \\
\hline 8 & 155 & OE1 & GLN & 18 & A & $<-$ & 2007 & $\mathrm{NZ}$ & LYS & 4 & B & 2.88 \\
\hline NEp6 & Atom no & Atom name & Res name & Res no & Chain & & Atom no & Atom name & Res name & Res no & Chain & Distance \\
\hline 1 & 52 & SD & MET & 6 & A & $<-$ & 1993 & $\mathrm{~N}$ & GLY & 3 & B & 3.08 \\
\hline 2 & 79 & $\mathrm{O}$ & PRO & 9 & A & $<-$ & 2010 & $\mathrm{NH} 2$ & $\mathrm{ARG}$ & 4 & B & 2.77 \\
\hline 3 & 105 & $\mathrm{O}$ & ALA & 12 & A & $<-$ & 2010 & $\mathrm{NH} 2$ & ARG & 4 & B & 2.74 \\
\hline 4 & 149 & $\mathrm{~N}$ & GLN & 18 & A & $->$ & 2050 & $\mathrm{O}$ & ASP & 8 & B & 3.31 \\
\hline NEp8 & Atom no & Atom name & Res name & Res no & Chain & & Atom no & Atom name & Res name & Res no & Chain & Distance \\
\hline 1 & 46 & $\mathrm{O}$ & SER & 5 & A & $<-$ & 2011 & NE2 & GLN & 4 & B & 3.1 \\
\hline 2 & 56 & $\mathrm{~N}$ & SER & 7 & A & $->$ & 2010 & OE1 & GLN & 4 & B & 2.78 \\
\hline 3 & 125 & $\mathrm{~N}$ & ALA & 15 & A & $->$ & 2022 & OE1 & GLU & 5 & B & 2.73 \\
\hline 4 & 131 & $\mathrm{~N}$ & ILE & 16 & A & $->$ & 2022 & OE1 & GLU & 5 & B & 2.73 \\
\hline 5 & 149 & $\mathrm{~N}$ & GLN & 18 & A & $->$ & 1992 & OE2 & GLU & 2 & B & 2.88 \\
\hline 6 & 156 & NE2 & GLN & 18 & A & $->$ & 1991 & OE1 & GLU & 2 & B & 2.77 \\
\hline 7 & 161 & $\mathrm{~N}$ & GLU & 19 & A & $->$ & 1992 & OE2 & GLU & 2 & B & 3.07 \\
\hline 8 & 188 & SD & MET & 21 & A & $<-$ & 1976 & $\mathrm{~N}$ & ILE & 1 & B & 3.34 \\
\hline
\end{tabular}

to an immunogenic peptide fused on its surface. Expression of PMV CP in bacterial cell like (E. coli) leads to assembly VLPs. C-terminal fusion is not always efficient it depends on the character of the epitope fused to the efficient platform. And demonstrated by taking CTL-epitope attained from the NP (NucleoCapsid) of the influenza virus, fused to the carboxyl-terminal region of the PMV CP, leading to nanoparticles presenting surface-Displayed epitope. While the Papaya Mosaic Viral is put forward as a vaccine platform is clearly flexible and shown to tolerate the fusion of several immunogenic peptides to its $\mathrm{C}$-terminus. This allows the displayed and expressed epitopes to freely adopt native conformations (Rioux et al. 2012).

Denis et al. reported earlier studied multimerization of the immunogenicity of Papaya Mosaic Viral like particles by fussing epitope of hepatitis-C virus and describe the engineering of PMV Capsid Protein as a carrier in addition to a Carboxyl-terminal fused with E2 epitope of hepatitis-C virus (Denis et al. 2007). The discovery of B-cell immunogenic epitopes is a crucial primary step for the advance of epitope peptide-based vaccines, diagnostic tools, and therapeutic antibodies. Immunogenic Epitope peptides can be displayed on the surface capsid protein subsequently those peptides assembled like virus particles act as vehicles for epitope display. Expression of immunogenic epitope peptides as fusions with surface loops of plant viral Capsid proteins has been used for the generation of several candidate animal vaccines (Hefferon 2018).

The Capripox viral envelop P32 protein multiple sequence alignment, amino acid sequences between each epitope and their homologous proteins showed that they were almost 100\% conserved among 11 P32 immunogenic sequences. The identified immunogenic epitope peptides are Ep3-110-118 regions of p32 envelop protein with peptide sequence ADIKNSENK, Ep6-regions between 11-19 of $\mathrm{p} 32$ protein with peptide sequence IVGREISDV and Ep8- 140-147 regions of p32 envelop protein with peptide sequence IEMQEKNI. These peptides are surface-displayed carboxyl and amino terminal regions of PaMV CP and identified the binding affinity each terminal regions. The binding capacity of immunogenic p32 epitope peptides with PaMV $\mathrm{CP}$ at Carboxy terminal (C) are EP3 $(-55.6 \pm 8.2)$, EP6 $(-62.0 \pm 5.2), \mathrm{EP} 8(-52.4 \pm 2.4)$ and the binding confirmation of epitope peptide displayed at C-terminus with Papayamosaic virus Capsid protein showed in Fig. 5. As well at amino-terminal $(\mathrm{N})$ of PaMV CP are EP3 $(-56.7 \pm 4.8)$, 
EP6 (- 55.4 \pm 2.0$)$ EP8( $-57.5 \pm 2.1)$ and the binding confirmation of epitope displayed at $\mathrm{N}$-terminus of Papayamosaic virus Capsid protein showed in Fig. 6. The three-dimensional structural modeled, identified analyzed epitopes were displayed on the surface of $\mathrm{N}$ and $\mathrm{C}$ terminals PMV CP. this will increase the perceptive of the P32 envelop protein structure and functions. And it may possibly place the foundation for the designing and development of a universal Capripox viral multi-epitope based peptide vaccine as well as detection antigen.

Acknowledgements The authors thanks REVA University, Bengaluru, Karnataka and ICAR-NIVEDI, Bengaluru, Karnataka for the support and facility to carry out this work.

\section{Compliance with Ethical Standards}

Conflict of interest The authors declare that they donot have conflict of interest.

Animal and Human Participants This article dose not contain any studies with animal or human subjects performed by the authors.

Informed Consent Authors stated that there is no informed consent in the article.

\section{References}

Altschul SF, Madden TL, Schäffer AA, Zhang J, Zhang Z, Miller W, Lipman DJ (1997) Gapped BLAST and PSI-BLAST: a new generation of protein database search programs. Nucleic Acids Res 25:3389-3402

Altschul SF, Wootton JC, Gertz EM, Agarwala R, Morgulis A, Schäffer AA, Yu YK (2005) Protein database searches using compositionally adjusted substitution matrices. FEBS J 272:5101-5109

Babin C, Majeau N, Leclerc D (2013) Engineering of papaya mosaic virus (PapMV) nanoparticles with a CTL epitope derived from influenza NP. J Nanobiotechnol 11:10

Bhanuprakash V, Indrani BK, Hosamani M, Singh RK (2006) The current status of sheep pox disease. Comp Immunol Microbiol Infect Dis 29:27-60

Bhavaniprasad V, Dassa JFP, Jayanthi S (2013) Activation mechanism of claudin-4 by ephrin type-A receptor 2: a molecular dynamics approach. Mol BioSyst 9:2627

Burranboina K, Abraham S, Murugan K, Bayyappa MR, Yogisharadhya R, Raghavendra G (2018) Genome wide identification and analysis of microsatellite repeats in the largest DNA viruses (Poxviridae Family): an insilico approach. Annu Res Rev Biol 22(1):1-11

Chen Q, Lai H (2013) Plant-derived virus-like particles as vaccines. Hum Vaccin Immunother 9(1):26-49

Crisci E, Bárcena J, Montoya M (2013) Virus-likeparticle based vaccines for animal viral infections. Inmunología 32(3):102-116

Denis J, Majeau N, Acosta-Ramirez E, Savard C, Bedard MC, Simard S, Lecours K et al (2007) Immunogenicity of papaya mosaic virus-like particles fused to a hepatitis $\mathrm{C}$ virus epitope: evidence for the critical function of multimerization. Virology 363:59-68

Dominguez C, Boelens R, Bonvin MJJA (2003) HADDOCK: a proteinprotein docking approach based on biochemical and/or biophysical information. J Am Chem Soc 125:1731-1737
Guex N, Peitsch MC (1997) SWISS-MODEL and the Swiss-PdbViewer: an environment for comparative protein modeling. Electrophoresis 18:2714-2723

Hefferon KL (2018) Repurposing plant virus nanoparticles. Vaccines 6:11

Idris ST, Salih S, Basheir M, Elhadi A, Kamel S et al (2018) In silico prediction of peptide based vaccine against Fowlpox Virus (FPV). Immunome Res 14:154

Ikram A, Zaheer T, Awan FM, Obaid A, Naz A, Hanif R, Paracha RZ, Ali A, Naveed AK, Janjua HA (2018) Exploring NS3/4A, NS5A and NS5B proteins to design conserved subunit multi-epitope vaccine against HCV utilizing immunoinformatics approaches. Sci Rep 8:16107

Kelley LA et al (2015) The Phyre2 web portal for protein modeling, prediction and analysis. Nat Protoc 10:845-858

Kumar S, Ochoa W, Singh P, Hsu C, Schneemann A, Manchester M, Olson M, Reddy V (2009) Tomato bushy stunt virus (TBSV), a versatile platform for polyvalent display of antigenic epitopes and vaccine design. Virology 388:185-190.

Kumar V, Damodharan S, Pandaranayaka EP, Madathiparambil MG, Tennyson J (2015) Molecular modeling and in-silico engineering of Cardamom mosaic virus coat protein for the presentation of immunogenic epitopes of Leptospira LipL32. J Biomol Struct Dyn 34(1):42-56

Laskowski RA, Jabłońska J, Pravda L, Vařeková RS, Thornton JM (2018) PDBsum: structural summaries of PDB entries. Protein Sci 27(1):129-134

Lovell SC, Davis IW, Arendall WB, de Bakker PIW, Word J, Prisant MG, Richardson JS, Richardson DC (2002) Structure validation by Calpha geometry: phi, psi and Cbeta deviation. Proteins 50:437-450

Madhavan A, Venkatesan G, Kumar A (2016) Capripoxviruses of small ruminants: current updates and future perspectives. Asian J Anim Vet Adv 11:757-770

Mhemid KM, Hassan IQ (2015) Molecular detection of lumpy skin disease virus in cattle by polymerase chain reaction in Iraq. Iraqi J Vet Med 40(1):83-88

Mirzaie K, Barani SM, Bokaie S (2015) A review of sheep pox and goat pox: perspective of their control and eradication in Iran. J Adv Vet Anim Res 2(4):373-381

Moming A, Tuoken D, Yue X, Xu W, Guo R et al (2018) Mapping of B-cell epitopes on the $\mathrm{N}$-terminal and C-terminal segment of nucleocapsid protein from Crimean-Congo hemorrhagic fever virus. PLoS ONE 13(9):e0204264

Naskalska A, Pyrc K (2015) Virus like particles as immunogens and universal nanocarriers. Pol J Microbiol 64(1):3-13

Nuzzaci M, Maria et al (2006) Cucumber mosaic virus as a presentation system for a double hepatitis $\mathrm{C}$ virus-derived epitope. Arch Virol 152:915-928

Porollo A, Meller J (2007) Prediction-based fingerprints of proteinprotein interactions. Proteins 66:630-645

Potocnakova L, Bhide M, Pulzova LB (2016) An introduction to B-cell epitope mapping and in silico epitope prediction. J Immunol Res. https://doi.org/10.1155/2016/6760830

Pushko P, Pumpens P, Grens E (2013) Development of virus-like particle technology from small highly symmetric to large complex virus-like particle structures. Intervirology 56(3):141-165

Rioux G, Babin C, Majeau N, Leclerc D (2012) Engineering of papaya mosaic virus (PapMV) nanoparticles through fusion of the HA11 peptide to several putative surface-exposed sites. PLoS ONE 7:e31925

Rostami A, Bashir NS, Pirniakan P, Masoudi N (2014) Expression of cucumber mosaic virus coat protein and its assembly into viruslike particles. Biotech Health Sci 1(3):e24729

Santi L, Huang Z, Mason H (2006) Virus-like particles production in green plants. Methods 40(1):66-76 
Shoeb E, Hefferon K (2019) Future of cancer immunotherapy using plant virus-based nanoparticles. Future Sci OA 5(7):FSO401

Tarello W, Kinne J (2007) Complete remission after treatment of Capripoxvirus infection in sheep using potassium arsenite $0.5 \%$ (Fowler's solution). Revue Méd Vet 158(10):489-492

Tuppurainen ESM, Venter EH, Shisler JL et al (2015) Review: Capripoxvirus diseases: current status and opportunities for control. Transbound Emerg Dis 64(3):729-745

van Zundert GCP, Rodrigues JPGLM, Trellet M, Schmitz C, Kastritis PL, Karaca E, Melquiond ASJ, van Dijk M, de Vries SJ, Bonvin AMJJ (2016) The HADDOCK2.2 webserver: user-friendly integrative modeling of biomolecular complexes. J Mol Biol 428:720-725
Varshovi HR, Norian R, Azadmehr A, Afzal Ahangaran N (2017) Immune response characteristics of Capripox virus vaccines following emergency vaccination of cattle against lumpy skin disease virus. IJVST 9:33-40

Zhou T, Jia H, Chen G, He X, Fang Y, Wang X, Guan Q, Zeng S, Cui Q, Jing Z (2012) Phylogenetic analysis of Chinese sheeppox and goatpox virus isolates. Virol J 9:25

Publisher's Note Springer Nature remains neutral with regard to jurisdictional claims in published maps and institutional affiliations. 